\title{
Melanocitoma meníngeo del ángulo pontocerebeloso: Claves imagenológicas para su abordaje
}

\author{
Javier Lara G. ${ }^{1}$, María José Sardá R. ${ }^{2^{*}}$, Luis Márquez B. ${ }^{3}$, Gerardo Sánchez R. ${ }^{2}$, Diana Michel C.
}

1. Médico Neurorradiólogo. Hospital de Especialidades del Centro Médico Nacional de Occidente del Instituto Mexicano del Seguro Social. Guadalajara, Jalisco, México.

2. Médico residente de tercer año de la especialidad en Imagenología Diagnóstica y Terapéutica. Centro Universitario de Ciencias de la Salud de la Universidad de Guadalajara. Guadalajara, Jalisco, México.

3. Médico residente de cuarto año de la especialidad en Imagenología Diagnóstica y Terapéutica. Centro Universitario de Ciencias de la Salud de la Universidad de Guadalajara. Guadalajara, Jalisco, México.

4. Médico residente de tercer año de la especialidad en Anatomía Patológica. Centro Universitario de Ciencias de la Salud de la Universidad de Guadalajara. Guadalajara, Jalisco, México.

\section{Cerebellopontine meningeal melanocytoma: Keys for imaging diagnostic approach}

\section{Resumen:}

Las lesiones del ángulo pontocerebeloso (APC) representan el 6 al 10\% de las neoplasias intracraneales, siendo los schwannomas vestibulares y meningiomas los más comunes. Sin embargo, hasta el 15\% pueden ser otras lesiones, entre ellas las derivadas a partir de restos de células melanocíticas presentes en las leptomeninges. El diagnóstico diferencial de las patologías tumorales del APC es extenso, siempre teniendo en cuenta las lesiones más comunes. Sin embargo, cuando las características radiológicas no son las esperadas, el enfoque debe orientarse hacia las lesiones inusuales, poniendo en contexto las diferentes estirpes celulares que pueden dar origen a las neoplasias en esta localización, como las neoplasias melanocíticas. Se presenta el caso de un masculino de 74 años con síndrome cerebeloso de tórpida evolución, al cual se le realiza RM de cerebro contrastada, identificando una lesión de base dural en el APC izquierdo, con hiperintensidad de señal en T1 e hipointensidad en T2, atípico para las lesiones más comunes en esta región, que sugiere su contenido melanocítico.

Palabras clave: Ángulo pontocerebeloso; Imagen por resonancia magnética; Melanina; Neoplasia.

\begin{abstract}
:
Cerebellopontine angle tumors (CPA) represent approximately 6 to $10 \%$ of intracranial tumors. Vestibular Schwannomas and meningiomas are the most common, however up to $15 \%$ can be of other origin, including from melanocytes derived from the neural crest. The differential diagnosis of CPA pathologies is extensive, always taking into account the most common ones. However, if the radiological characteristics are not the expected, the approach should be directed towards unusual lesions, putting into context the different cell lines that can give rise to the neoplasm at this location, such as melanotic neoplasms. We present a case of a 74-year-old male, who presented with a cerebellar syndrome. Due to an atypical clinical evolution, a contrast enhanced head MRI was performed, revealing a dural based tumor on the left CPA, which was hyperintense on T1 and hypointense on T2 weighted sequences, which is not expected from the common lesions at this region and suggested it's melanotic content.
\end{abstract}

Keywords: Cerebellopontine angle; Magnetic resonance imaging; Melanin; Neoplasm.

Lara J. et al. Melanocitoma meníngeo del ángulo pontocerebeloso: claves imagenológicas para su abordaje. Rev Chil Radiol 2020; 26(3): 113-116.

*Correo electrónico: Maria José Sardá Ramírez / majose.sarda@gmail.com

Trabajo enviado el 15 de abril de 2020. Aceptado para publicación el 03 de julio de 2020. 


\section{Introducción}

Las lesiones del ángulo pontocerebeloso (APC) representan aproximadamente el 6 al 10\% de las neoplasias intracraneales, las cuales debido a las diversas estructuras del APC (nervios, arterias, líquido cefalorraquídeo, meninges y sus precursores embriológicos), pueden ser de naturaleza muy variable ${ }^{1}$. Los Schwannomas vestibulares y los meningiomas son las dos lesiones más frecuentes, abarcando del 80 al $90 \%$ de la totalidad, mientras que los quistes epidermoides se sitúan en el tercer lugar, representando el $5 \%$. Otras lesiones, correspondientes del 1 al $15 \%$ del total de los tumores en esta localización, presentan un espectro amplio de hallazgos por imagen que vuelven un reto su caracterización diagnóstica ${ }^{1,2}$.

Los melanocitomas meníngeos son tumores primarios poco frecuentes, descritos por primera vez hace poco más de 30 años $^{3}$, cuya incidencia anual se ha estimado en 1 por cada 10 millones. Se desarrollan a partir de restos de células melanocíticas derivadas de la cresta neural presentes en las leptomeninges ${ }^{1}$. Su edad de presentación alcanza un pico máximo durante la cuarta y quinta décadas de la vida; su localización más habitual es intradural extramedular en la región cervical, seguida de la base del cráneo y por último en el ángulo pontocerebeloso (APC), donde existen reportes de alrededor de 22 casos ${ }^{1}$.

\section{Descripción}

Presentamos el caso de un paciente masculino de 74 años de edad, que desarrolla un cuadro clínico con marcha atáxica, vértigo y lateralidad izquierda, completando así un síndrome cerebeloso. Fue diagnosticado con síndrome de Ménière siete años antes por presentar hipoacusia, vértigo y nauseas, el cual fue tratado con betahistina, con mejoría parcial. Debido a su tórpida evolución y a su sintomatología agregada, se decide realizar resonancia magnética (RM) contrastada de cerebro, en donde se identificó una lesión extra-axial y de base dural en el APC izquierdo, que determinaba desplazamiento cerebeloso, con hiperintensidad en T1 (Figura 1) e hipointensidad en T2 (Figura 2) y reforzamiento tras la administración de gadolinio (Figura 3).

Se realizó resección parcial de la lesión y la histología demostró una neoplasia con diferenciación melanocítica sin atipia (Figura 4), que en el análisis inmuno-histoloquímico demostró positividad para anticuerpos proteína S-100 y anti-melanoma (HMB-45), mientras que el Ki67 presentó positividad nuclear en 3\% (Figura 5); estableciendo diagnóstico definitivo de melanocitoma meníngeo.

Luego de la resección parcial, se decide someter al paciente a radioterapia adyuvante, presentando importante mejoría clínica y reducción tumoral de 2 $\mathrm{mm}$ en todos sus ejes. Posteriormente es enviado a rehabilitación, continuando vigilancia por parte del servicio de neurocirugía de nuestra unidad hospitalaria.

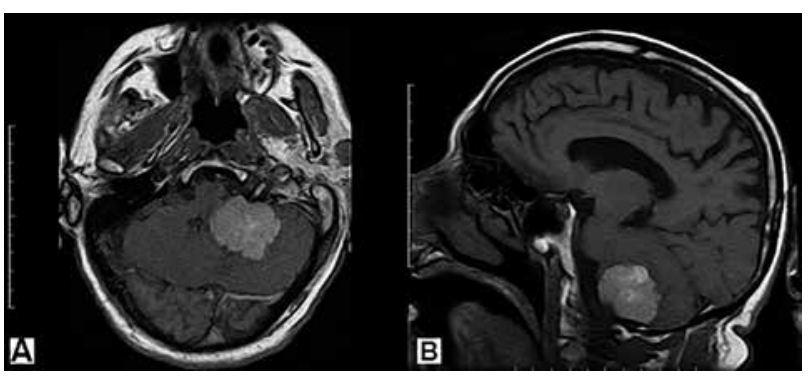

Figura 1: Secuencia T1 en fase simple en plano axial (a) y sagital (b), con presencia de una lesión extra-axial localizada en el APC izquierdo, de base dural y con bordes bien delimitados, que se comporta hiperintensa.

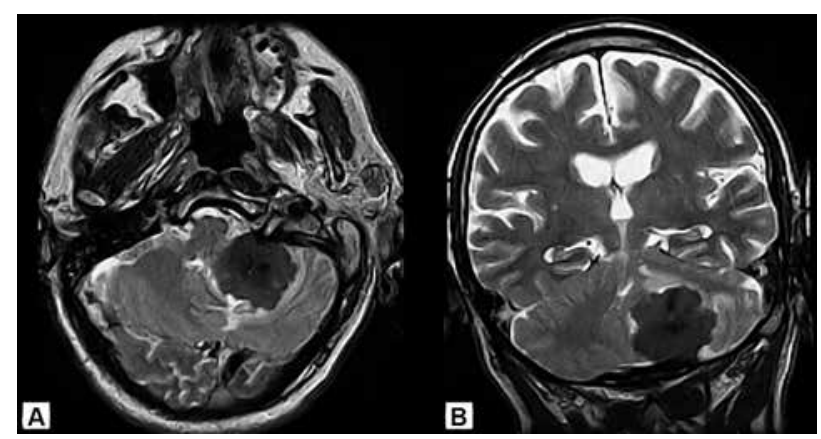

Figura 2: Secuencia T2 en plano axial (a) y coronal (b) que evidencia una importante hipointensidad de la lesión en el $A P C$ izquierdo.

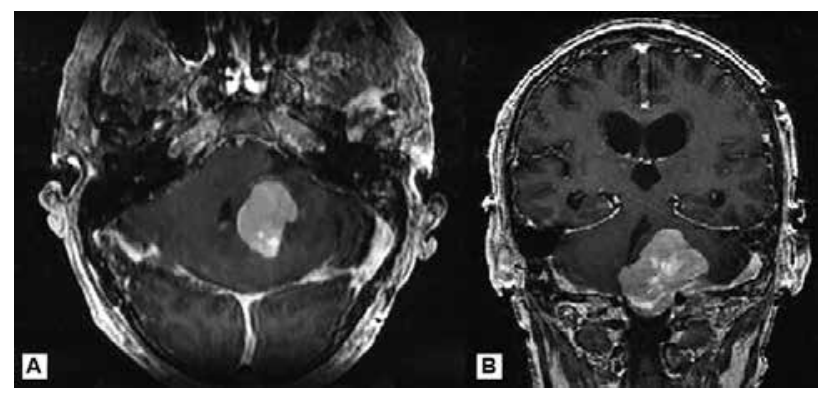

Figura 3: Plano axial (a) y coronal (b) en secuencias T1 posterior a la aplicación de gadolinio, que demuestra reforzamiento de la lesión. 


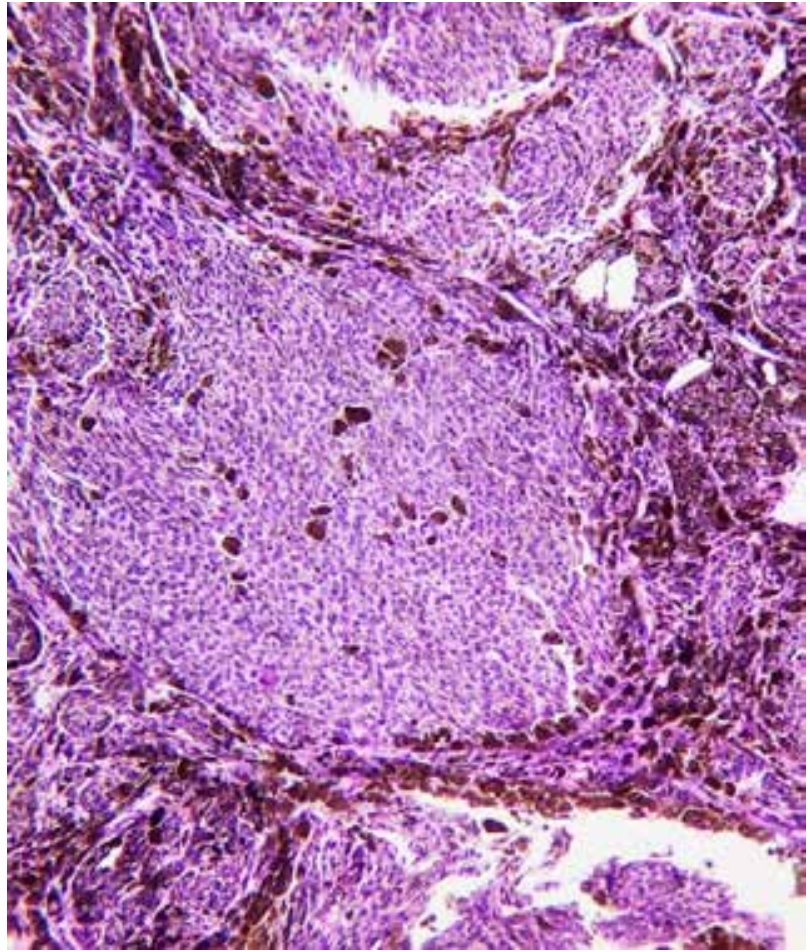

Figura 4: Tinción H\&E. Vista panorámica de neoplasia melanocítica con crecimiento en nidos sólidos, delimitados por septos fibrosos finos.

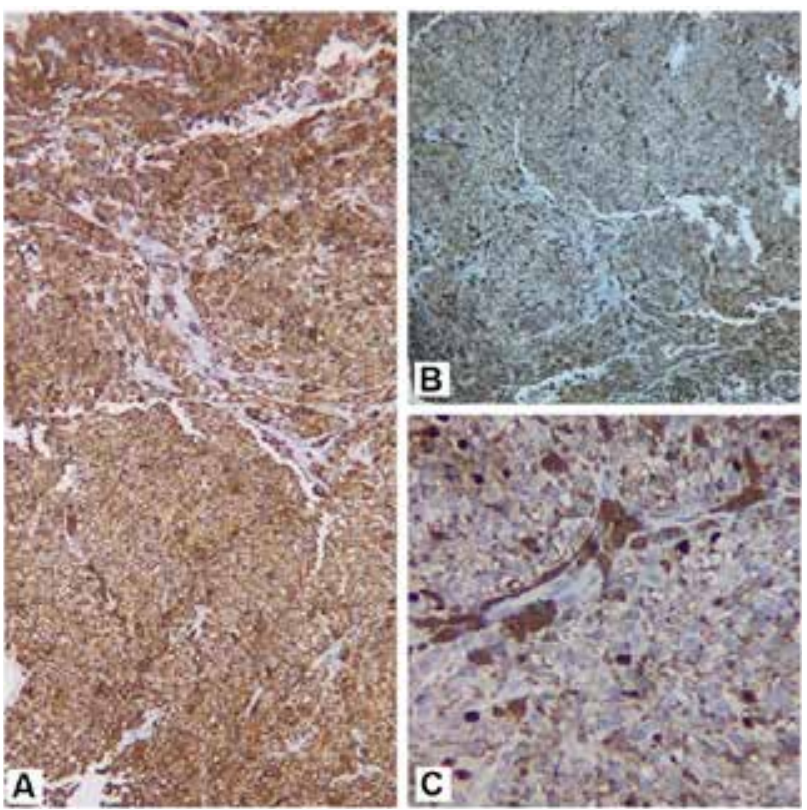

Figura 5: Imagen 3: Inmunorreactividad positiva para HMB-45 (a) y $S 100$ (b) que confirman el diagnóstico. Ki67 (c) muestra índice de proliferación de $3 \%$.

\section{Discusión}

Las neoplasias melanocíticas del sistema nervioso central (SNC) son raras, siendo el origen metastásico lo más frecuente ${ }^{2}$. Sin embargo, pueden presentarse lesiones primarias del SNC a partir de los melanocitos leptomeníngeos de origen neuroectodérmico que predominan en la base del cráneo y la superficie ventral de la médula espinal, condicionando patrones de crecimiento difuso que originan melanosis neurocutánea, melanomatosis leptomeníngea primaria o nodular, melanocitoma meníngeo y el melanoma ${ }^{2,4,5}$. La melanina contenida en el citoplasma celular les brinda propiedades relacionadas con radicales libres paramagnéticos (semiquinonas e indol semiquinonas), cuyos electrones no apareados interactúan con los protones del agua, resultando en atracciones dipolo-dipolo que producen acortamiento del T1 y T2 en la resonancia magnética².

El melanocitoma leptomeníngeo generalmente se presenta como una lesión tumoral solitaria extraaxial, de márgenes bien definidos, con una alta intensidad de señal en T1 e hipointensidad en T2, con variaciones de acuerdo al contenido de melanina $^{3}$; asociado con un realce ávido y homogéneo tras la administración de gadolinio $0^{1,2,3,5}$. Histopatológicamente, son neoplasias de bajo grado que no suelen infiltrar las estructuras adyacentes ${ }^{4,5}$, que pueden ser confundidos con meningiomas debido a su ubicación extra-axial y que a la microscopía presentan nidos celulares muy poblados (Figura $4)^{4}$. Sin embargo, el análisis inmunohistoquímico demuestra expresión de proteínas marcadoras melanocíticas (como S-100, melan-A y HMB-45) que ayudan a distinguir su naturaleza celular (Figura $5)^{3,4,6}$. La mayor distinción frente al melanoma es la escasa actividad mitótica, pleomorfismo nuclear e hipercromacia nuclear, así como su patrón de crecimiento indolente 4 .

El diagnóstico diferencial de las patologías tumorales del APC es extenso, más aún si las características por imagen no son las esperadas para las entidades que se presentan con mayor frecuencia en esta región. Ante este escenario, el enfoque debe orientarse hacia las lesiones inusuales, poniendo en contexto las diferentes estirpes celulares que pueden dar origen a las neoplasias en esta localización, en conjunto con su comportamiento en RM, para así reducir las posibilidades en el diagnóstico diferencial. Aunque raras, las neoplasias melanocíticas pueden presentarse en el APC, mostrando hallazgos típicos en imágenes que, combinados con el entorno clínico, pueden orientar hacia el diagnóstico'.

En el presente caso, el comportamiento de la lesión, hiperintenso en T1 y marcadamente hipointenso en secuencias T2 (Figuras 1 y 2), sugiere 
contenido paramagnético, lo que ayuda a dirigir el diagnóstico hacia lesiones melanocíticas y permite diferenciarlo de otras que se presentan de forma clásica en topografía del APC, como el schwannoma y meningioma, que de manera habitual son hipointensos en T1 e hiperintensos en T2. No se debe olvidar que algunas lesiones del APC pueden presentar contenido pigmentario y simular la apariencia de una neoplasia melanocítica, entre ellas cabe mencionar las variedades melanóticas del schwannoma y el meduloblastoma. Además, existen otras lesiones que pueden presentar hiperintensidad en T1 debido a su contenido lipídico, como los quistes dermoides y los lipomas, así como los poco frecuentes quistes epidermoides blancos, que al presentarse iso o hiperintensos en T2, pueden ser diferenciadas de aquellas con contenido melanocítico ${ }^{1,4}$. Es en este escenario donde las características por RM y la confirmación anatomopatológica cobran un papel muy importante en el abordaje. En nuestro paciente, que muestra hallazgos imagenológicos compatibles con una lesión pigmentada del ángulo pontocerebeloso, el análisis histopatológico demuestra baja tasa de replicación celular en las tinciones específicas (Ki67), y además evidencia la presencia de melanina en el tejido neoplásico tanto en la tinción de H\&E como en la inmunohistoquímica siendo positivo para HMB-45, lo que establece el diagnóstico de melanocitoma.

\section{Referencias}

1. Bonneville F, Sarrazin JL, Marsot-Dupuch K, Iffenecker C, Cordoliani Y-S, Doyon D, et al. Unusual Lesions of the Cerebellopontine Angle: A Segmental Approach. RadioGraphics. 2001; 21(2): 419-438.

2. Bonneville F, Savatovsky J, Chiras J. Imaging of cerebellopontine angle lesions: An update. Part 1: Enhancing extra-axial lesions. Eur Radiol. 2007; 17(10): 2472-2482.

3. Jaiswal S, Vij M, Tungria A, Jaiswal AK, Srivastava AK, Behari S. Primary melanocytic tumors of the central nervous system: A neuroradiological and clinicopathological study of five cases and brief review of literature. Neurol India. 2011; 59(3): 413419.

4. Smith $A B$, Rushing EJ, Smirniotopoulos JG. From the archives of the AFIP pigmented lesions of the central nervous system: Radiologic-pathologic correlation. Radiographics. 2009; 29(5): 1503-1524.

5. Liubinas S V., Maartens N, Drummond KJ. Primary melanocytic neoplasms of the central nervous system. J Clin Neurosci. 2010; 17(10): 1227-1232. Available from: http://dx.doi.org/10.1016/j.jocn.2010.01.017

6. Lee PH, Wang LC, Lee EJ. Primary intracranial melanoma. J Cancer Res Pract. 2017; 4(1): 23-26. Available from: http://dx.doi.org/10.1016/j.jcrpr.2016.09.001 